\title{
Confirm Content Validity and Sender Authenticity for Text Messages by Using QR Code
}

\author{
Firas Mohammed Aswad \\ Yasir Ali Matni \\ Inteasar Esmaeel Khudair Ahmed Ehsan Mohammed \\ University of Diyala, College of Basic Education \\ Altae13@yahoo.com_ee22a12@gmail.com \\ Inteasar_yassin@yahoo.com \\ assel_child@yahoo.com
}

Keywords: Electronic messages, message's symbols, Digital signature, ASCII code, QR Code \#,C language

\begin{abstract}
In light of the information revolution taking place in the modern world, therefore it becomes necessary and important to save this electronic messages. So we offered this technique to ensure the safety of the content of the messages and authenticity of the sender through networks communication by converting the message's symbols to numbers, each one of this symbols (letters, numbers, symbols) will converted into three digits, the first digit represents the ASCII code of the symbol, the second digit represents the frequency of this symbol in the message (the number of times this symbol is appear in the message), and the third digit represents the total number of the locations of the symbol (calculates the symbol location from the first symbol in the message to this symbol itself and blanks also calculated too).The digital signature of the sender will converted to numbers like the symbols of message we explained it before, and this numbers of the digital signature will gathering together to produce three numbers only, this number will gathering with each numbers of the message's symbols, the final numbers will converted to QR Code, the QR Code will placed with the message and sent to the recipient. The recipient returns the steps of the sender (produce QR Code from the received message) and compared it the received QR Codes, if it is match or not. The recipient will ensure that the content is secure, and confirms the authenticity of the sender.
\end{abstract}




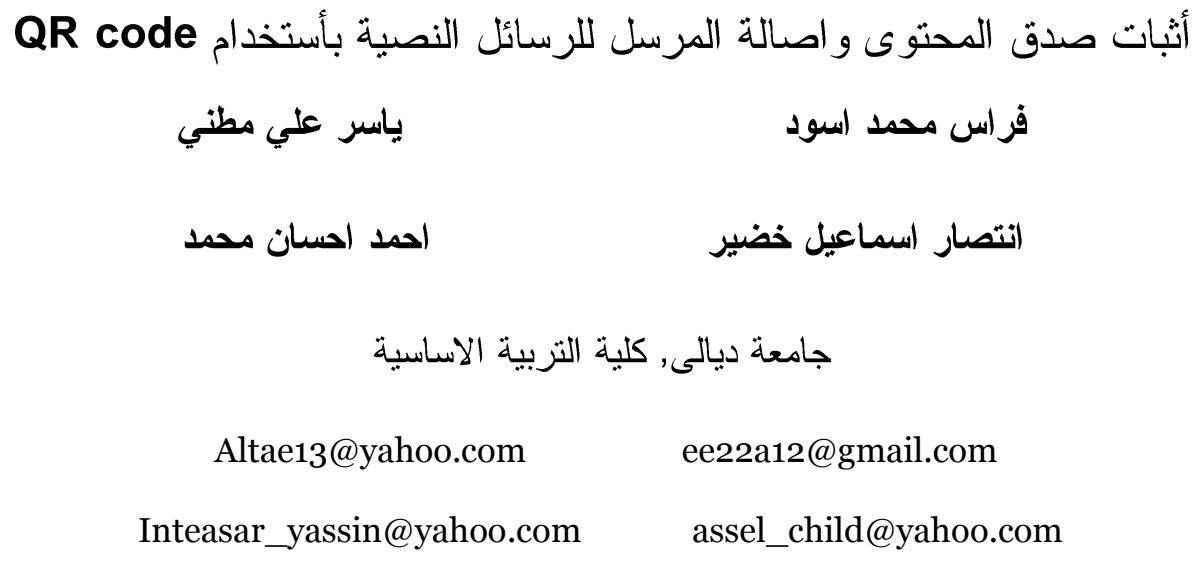

الخلاصة

في ظل ثورة المعلومات التي يشهدها عالمنا الحديث,اصبحت المراسلات الالكترونية ضرورية ومن المهم حفظ هذه المعلومات المرسلة. لذلك عرضنا هذه التقنية لضمان سلامة محتوى الرسائل و أصالة المرسل عبر شبكات الاتصالات عن طريق تحويل رمز الرسالة إلى أرقام، كل واحد من رموز الرسالة (الحروف والأرقام والرموز) سوف تحول إلى ثناثة أرقام، الرقم الأول يمثل أسكي كود الرمز، و الرقم الثاني يمثل تردد هذا الرمز في الرسالة (عدد المرات التي يظهر فيها هذا الرمز في الرسالة)، و والرقم

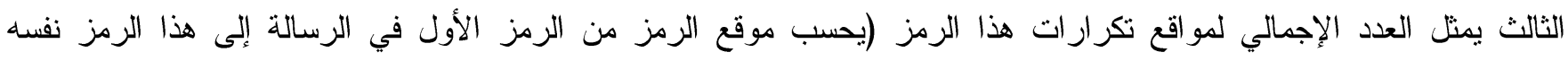

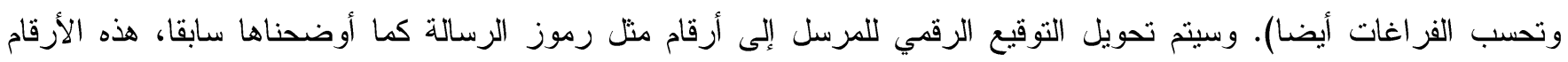

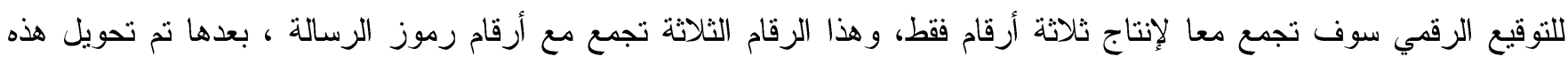

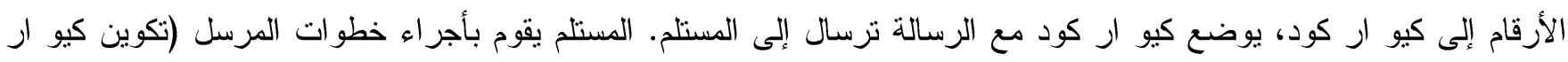
كود من الرسالة المستلمة) ويتم مقارنة الكيو ار كود ما إذا كان مطابق أم لا. وسيضمن المستلم أن المحتوى آمن، ويؤكد صحة المرسل.

$$
\text { الكلمات المفتاحية: رسائل الكترونية, رموز الرسالة, النوقيع الرقمي, الاسكي كود, الكيو ار كود , لغة سي\#. }
$$

المقدمة

يمثل أمن وسرية المعلومات حماية وتأمين الموارد المستخدمة كافة والعمل على سريتها وسالمتها , وفي غياب أمن

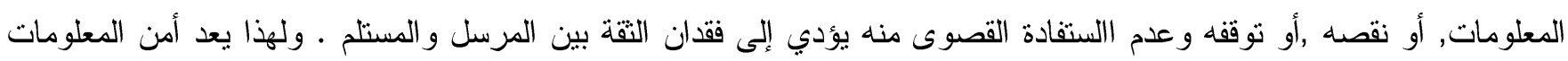
من الركائز الضرورية والحاكمة في حماية الأفراد والثركات والحكومات من الاضرار الناتجة ,ولضمان أمن المعلومات وسريتها هنالك طرق دقيقة وملائمة وموثوق منها رمثل الجدران النارية وكلمة السر والتثفير وغيرها من الطرائق التي تستخدم لعدم إفثاء البيانات و المعلومات المخزونة و المرسلة [1]]. 
مع تعاظم دور شبكات المعلومات في عالمنا ، و ازدياده حجم تبادل البيانات و المعلومات عبرها، واحتو اء الثبكة على كم هائل من المعلومات الهامة جدا، أصبحت عملية حماية تلك البيانات الهامة احد أهم التحديات التي يسعى المتخصصون في مجال حماية المعلومات توفير ها. وتنين الدراسات وجود تز ايد ملحوظ في عمليات اختراق تلك البيانات الهامة، مما يتطلب صباغة وتطوير طرق جديدة لحماية تلك المعلومات التي لا تقدر بثمن، وكذلك حماية وتأمين كافة المعلومات الرقمية , ومن وسائل الحماية تلك ظهور مفهوم التوقيع الرقمي Digital Signature.

الغش والخداع من الهجمات الامنية التي تواجه عملية تتاقل الرسائل النصية والمستندات والوثائق عبر شبكة الانترنيت وخصوصا مؤسسات الحوكمة الالكترونية والتجارة الالكترونية والتعاملات المالية الالكترونية حيث ان الغش و الخداع لله عدة اوجه منها تزييف المعلومات , تحويرها , تغيير المحتوى , كذلك انكار المسؤولية بالارسال من قبل المرسل الاصلي او الادعاء بان يكون المتطفل هو الطرف المرسل المخول [2].

عناصر أمن المعلومات

السرية Confidentiality: واحدة من الطرق التي نحافظ فيها على سرية المعلومات هي بالتشفير . التكاملية Integrity: تعبر عن كيف نقوم بتخزين البيانات ونتأكد بأنه لم يتم التعديل عليها سواء من قبل المخترقين أو حتى الأشخاص المصرح لهم بالاطلاع على البيانات. وكذللك عند نقل البيانات من مكان لمكان فهل تم اعتر اض هذه البيانات والتعديل عليها؟

التو افر Availability: و هي أن تكون البيانات أو الأجهزة و الثبكات متوفرة عند طلبها وعند الحاجة إليها [4].تم استخدام الباركود على نطاق واسع لتحديد اصلالة المنتجات , رموز الاستجابة السريعة (كيو ار كود) تمثل ببعدين من الباركود التي يمكن ان تتضمين نص ,صوت , فيديو, عنوان على شبكة الإنترنت، ووثائق التقويض وأكثر من ذلك بكثير. تتاول بعض البحوث مفهوم الكيو ار كود و استخداماتهة في ترميز الرسالئل , لقد اقترح تشفير بيانات باستخدام خوارزمية التشفير (AES). ويستند العمل تحويل الرموز المشفرة إلى كيو ار كود و من ثم باستخدام الماسح الضوئي لفك تثفيرها [5].

ان اهمية هذا البحث تكمن في الحفاظ على محتوى الرسائل واصالة المرسل لهذه الرسائل , ولاهمية هذه الرسائل التي من الممكن ان تكون سرية وذات اهمية كبيرة مثل التخاطبات الحكومية الاككترونية والمعاملات المالية والتجارية, هذه التقنية التي تم تصميمها تعمل وبشكل مثالي الى حد ما على التاكد من سلامة محتوى الرسائل المرسلة في الثبكات و الاتصالات و اصالة المرسل, و وان الهدف من البحث هو اثبات صدق وسلامة محتوى الرسائل من التحوير والتلاعب و التزييف وكذللك اثبات اصالة المرسل , وعدم الانكار بالنسبة للمرسل المخول[3]. 
نقدم هنا في بحثا هذا امكانية التاكد من سلامة محتوى الرسائل واصالة مرسلها بشكل مثالي الى حد ما, عن طريق تمثيل ثلاثة ارقام لكل رمز من رموز الرسالة ان كان حرف او رقم او رمز معين, فالرقم الاول يمثل الاسكي كود(ASCII code هو نظام ترميز من V بت يستخدم سبعة أرقام ثنائية القاعدة (قيمة تتر اوح بين · و Y I ) لتمثيل الحروف و الارقام و الرموز كما موضح في الشكل (1-1) هناك اهية لهذا الرمز ففي حال تغير هذا الرمز بفعل منطفل فسيتغير هذا الرقم الاول, اما الرقم الثاني فيمثل تكرار هذا الرمز في الرسالة كذلك سيتغير هذا الرقم اذا تغير رمز واحد من هذه الرموز واما الرقم الثالث وهو مهم جدا فهو يمثل مو اقع تكر ار ات هذا الرمز في الرسالة ففي حال نم تقديم وتاخير اي رمز من رموز الرسالة سيتغير هذا الرقم.

\begin{tabular}{|c|c|c|c|c|c|c|c|c|c|c|c|}
\hline Dec & Char & Dec & Char & Dec & Char & Dec & Char & Dec & Char & Dec & Char \\
\hline 33 & $!$ & 49 & 1 & 65 & A. & 81 & Q & 97 & $\mathrm{~s}$ & 113 & q \\
\hline 34 & $"$ & so & 2 & 66 & B & 82 & $\mathrm{R}$ & 98 & b & 114 & I \\
\hline 35 & H & 51 & 3 & 67 & c & 83 & $s$ & 99 & c & 115 & $s$ \\
\hline 36 & $\$$ & 52 & 4 & 68 & D & 84 & $\mathrm{~T}$ & 100 & d & 116 & $t$ \\
\hline 37 & $\%$ & 53 & 5 & 69 & $E$ & 85 & $U$ & 101 & e & 117 & $\mathrm{u}$ \\
\hline 38 & \&: & 54 & 6 & 20 & F & 86 & $\mathrm{~V}$ & 102 & $f$ & 118 & $\mathrm{v}$ \\
\hline 39 & . & sS & 7 & 71 & G & 87 & $w$ & 103 & $g$ & 119 & $w$ \\
\hline 40 & $c$ & 56 & 8 & 72 & $\mathrm{H}$ & 88 & $x$ & 104 & $\mathrm{~h}_{\mathrm{L}}$ & 120 & $x$ \\
\hline 41 & 3 & 57 & 9 & 73 & I & 89 & $Y$ & 105 & $i$ & 121 & $y$ \\
\hline 42 & * & 58 & $:$ & 74 & $\mathrm{~J}$ & 90 & $z$ & 106 & $j$ & 122 & $\mathrm{x}$ \\
\hline 43 & + & 59 & ; & 75 & $\mathrm{~K}$ & 91 & I & 107 & $\mathrm{k}$ & 123 & 6 \\
\hline 44 & , & 60 & $<$ & 76 & L & 92 & $i$ & 108 & 1 & 124 & 1 \\
\hline 45 & - & 61 & $=$ & 77 & $\mathbf{M}$ & 93 & ] & 109 & $m$ & 125 & 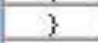 \\
\hline 46 & . & 62 & $=$ & 78 & $\mathrm{H}$ & 94 & $\therefore$ & 110 & $\mathbf{n}$ & 126 & $\sim$ \\
\hline 47 & 7 & 63 & $?$ & 79 & 0 & 95 & - & 111 & 0 & 127 & \\
\hline 48 & 0 & 64 & Q & 80 & P & 96 & . & 112 & p & & \\
\hline
\end{tabular}

الشكل (1- (1) (1) (1) (1) (1)

ملاحظة هذه التقنية تدعم الرسائل باللغة الانكليزية و العربية.

خوارزمية أثبات صدق المحتوى واصالة المرسل للرسائل النصية بأستخدام QR code

اولا: من جاتب المرسل

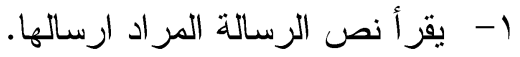

ץ- يمثل كل رمز من رموز الرسالة (احرف, ارقام,رموز) بثلاث ارقام الرقم الاول يمثل الاسكي كود لهذا الرمز, والرقم الثاني يمثل تكرار هذا الرمز في الرسالة(عدد مرات تو اجد هذا الرمز بالرسالة), اما الرقم الثالث فيمثل مجموع مواقع تكرارات هذا الرمز ( يحسب موقع الرمز من اول رمز وصو لا له و الفر اغات تحسب كذلك). 
س- يرمز التوقيع الرقمي (المعلوم لدي المرسل والمستلم) بنفس الطريقة في الخطوة الثانية(ابي لكل رمز من رموز التوقيع الرقمي يمثل بثلاثة ارقام), ويجمع كل قيم هذه الرموز ليكون هناك ثلاثة ارقام للتوقيع الرقمي(الرقم الاول هو مجموع الاسكي كود لكل رمز من رموز التوقيع الرقميوالرقم الثاني يمثل مجموع تكرارات رموز التوقيع الرقمي,والرقم الثالث

$$
\text { يمثل مجموع مواقع تكرارات رموز التوقيع الرقمي). }
$$

ع- يجمع ارقام كل رمز من رموز الرسالة المراد ارسالها (الناتجة من الخطوة الثانية) مع ارقام التوقيع الرقمي(لناتجة من

الخطوة الثالثة).

$$
\text { ث- تانيا: من جول هذه القيم الناتجة في الخطوة الر ابعة الى QR Code ويرفق مع الرسالة ويرسل الى المستلم. }
$$

1- يقوم المستلم بتتحويل رموز الرسالة المستلمة الى QR Code كما في جانب المرسل. r- بطابق ال QR Code المنتج من رموز الرسالة و ال QR Code المستلم مع الرسالة. ب- اذا كانت النتيجة مطابقة فهذا دليل على سلامة محتوى الرسالة واصالة مرسلها, اما اذا كانت النتيجة غير منطابقة فهذا دليل على ان هناك تلاعب او تزييف او تغيير في محتوى الرسالة او عدم مصداقية مرسلها. اذا هذه التقنية تكثف اذا تم اضافة او حذف اي رمز من رموز الرسالة وتكثف كذلك اذا تم تغيير ترتيب رموز الرسالة فمثلا لو ارسل رقم (value) وتم تغيره بفعل متطفل الى (valeu) سيتم كثف هذا التغيير من خلال هذه الطريقة كما سيتبين ذلك اكثر في المر احل الاخرى من بحثنا هذا.

تم برمجة هذه الخوارزمية بلغة (\#C) سي \# (بالإنجليزية: C\#) (تلفظ سي شارب) هي لغة برمجة متعددة الأنماط تتمتع بكونها سكونية التنميط و أمرية وتعريفية ووظيفية وإجرائية وعمومية وشيئية المنحى (غرضية التوجه) (باستخدام الصفوف) كما تخضع لمبادئ البرمجة التركيبية المنحى [6]. ملاحظة: كود البرمجة بلغة \#C فقط. مثال عملي مثال عملي على ما تقدم من ذكره موضح بالصور و الثرح بالتفيل, منل ندخل كلمة (VALUE) نكتب نص كلمة (VALUE) في المربع المخصص له ونضغط على مفتاح (استدعاء النص), حيث سيتم حساب الاسكي كود لكل حرف, وان التوقيع الرقمي هو (computer) 


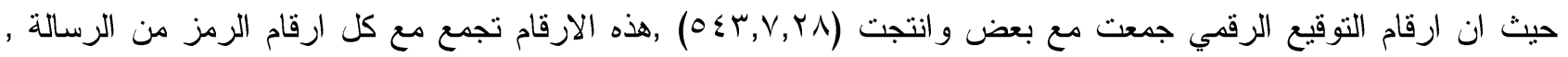

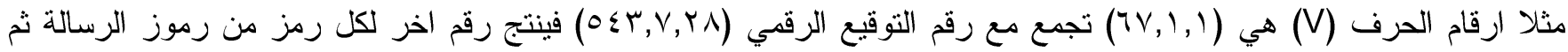
تحول هذه الارقام الاخيرة الى كيو ار كود.

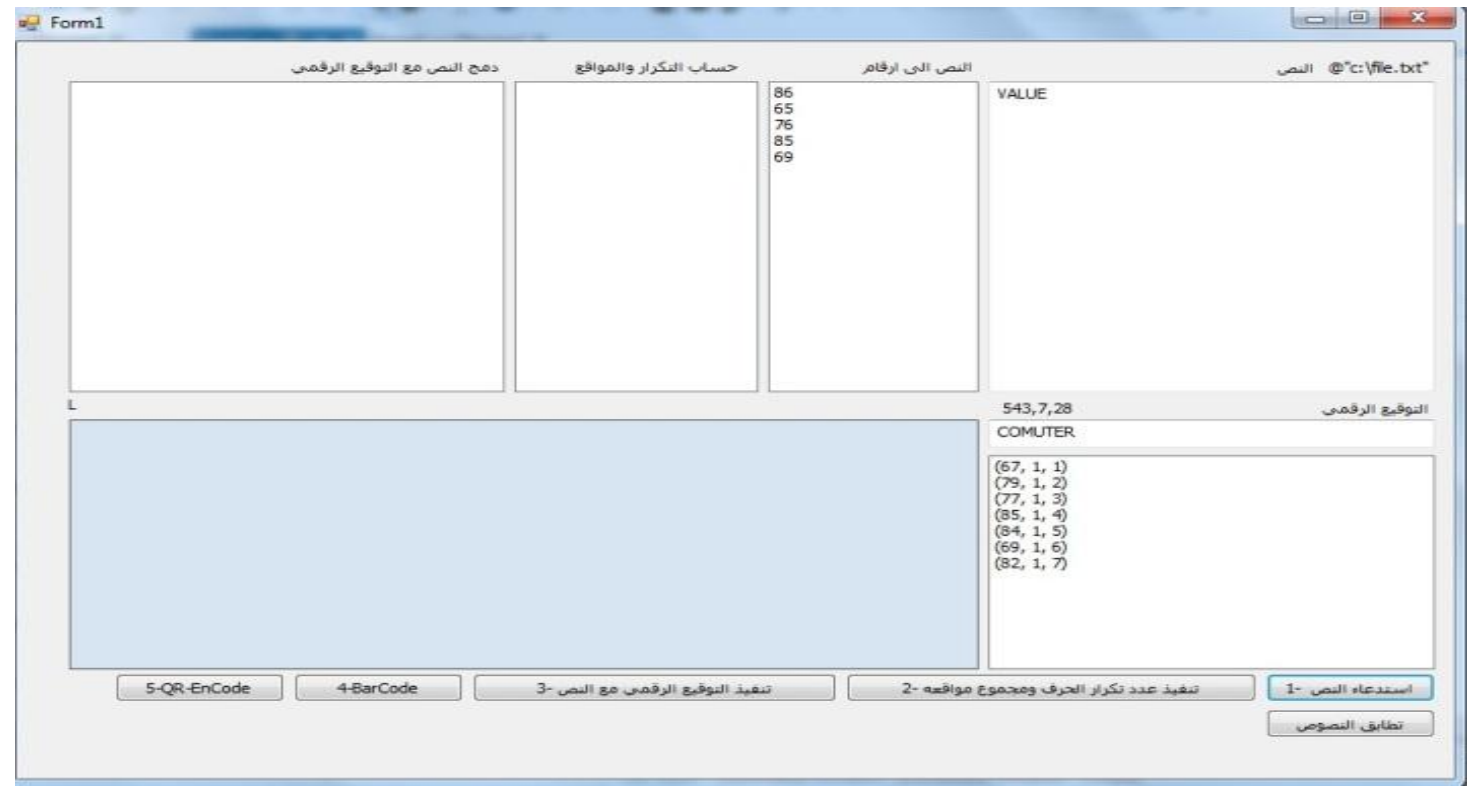

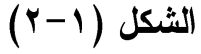

بعدها نضغط على مفتاح (تنفيذ عدد نكرار الحرف ومجموع مو اقعه), حيث سينم حساب تكرار كل حرف وحساب مجموع مو اقع تكر اراته كما في الثكل(1-1)

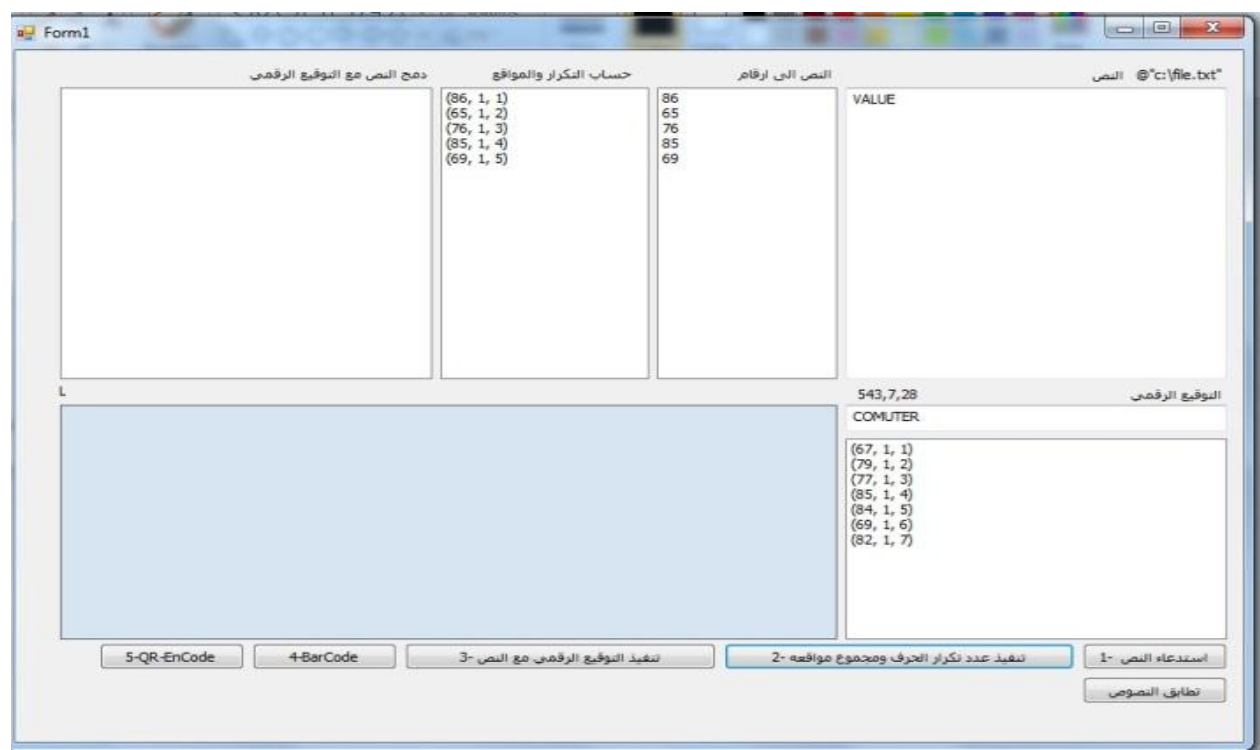

الثكل(1- 
Journal of University of Babylon for Pure and Applied Sciences,Vol.(26), No.(7): 2018

وبعد هذه الخطوة نضغط على مفتاح QR-EnCode لتكوين QR-Code خاص لهذه الكلمة المكتوبة كما في الثكل (ع-1)

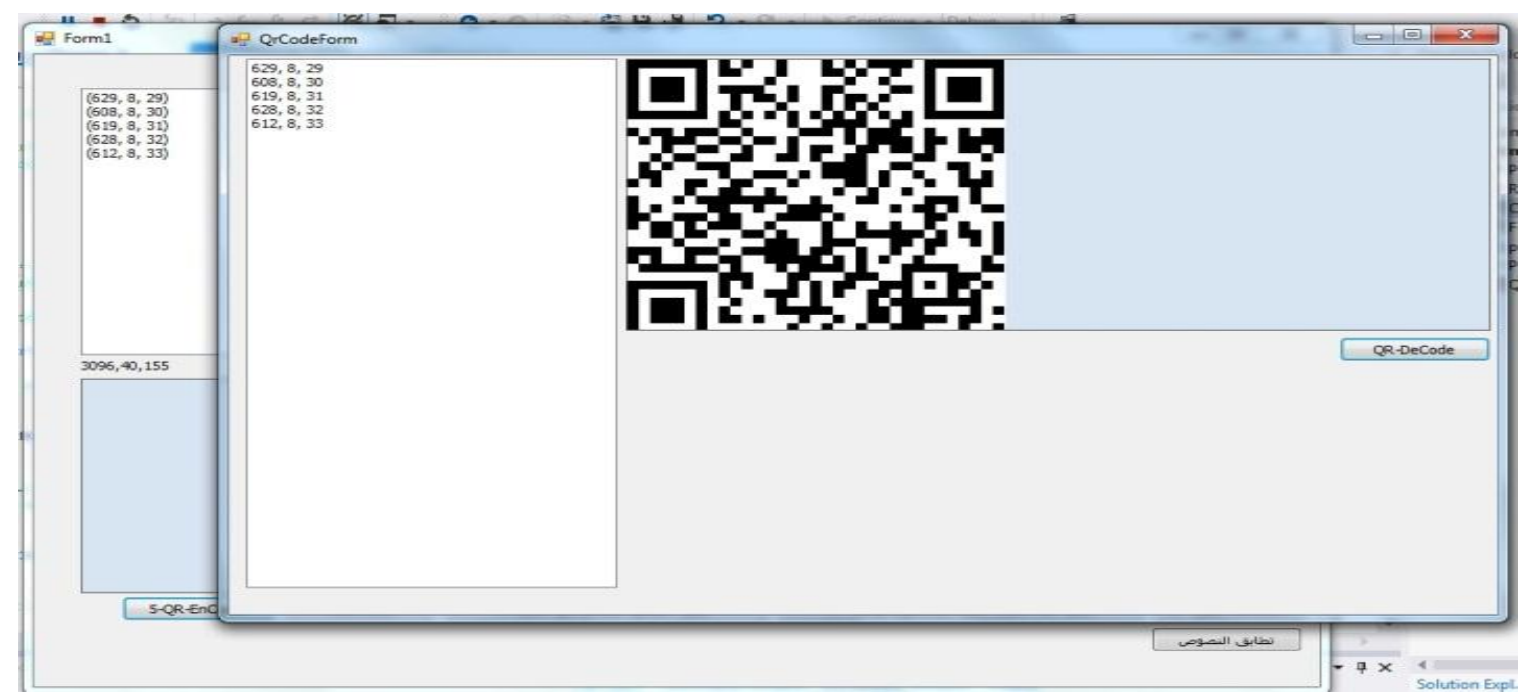

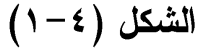

الان نعيد الخطوات السابقة ولكن بتغيير مواقع حرفين مثل (VALUE) كما في الاشكال التالية (الثكل (0-1), الثكل (؟-1),

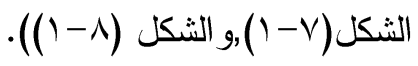

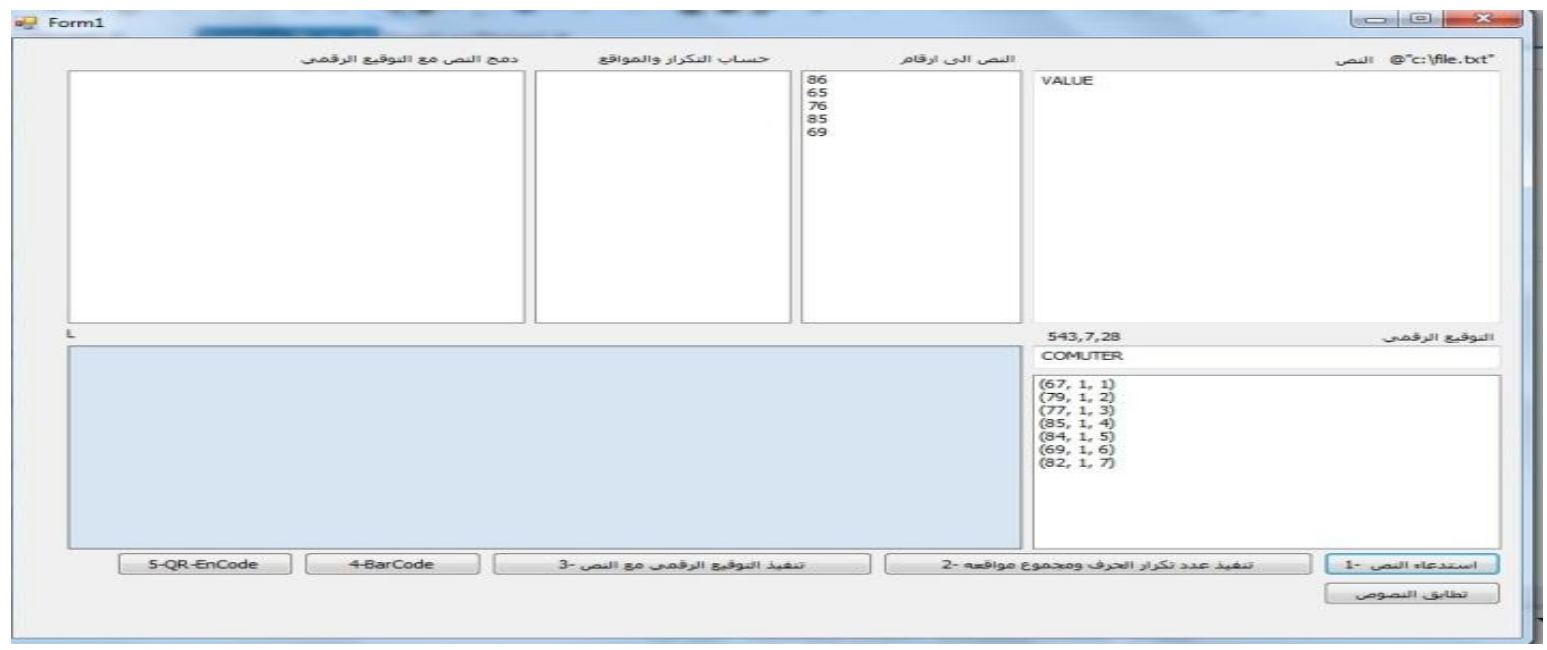

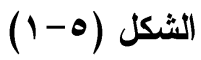


Journal of University of Babylon for Pure and Applied Sciences,Vol.(26), No.(7): 2018

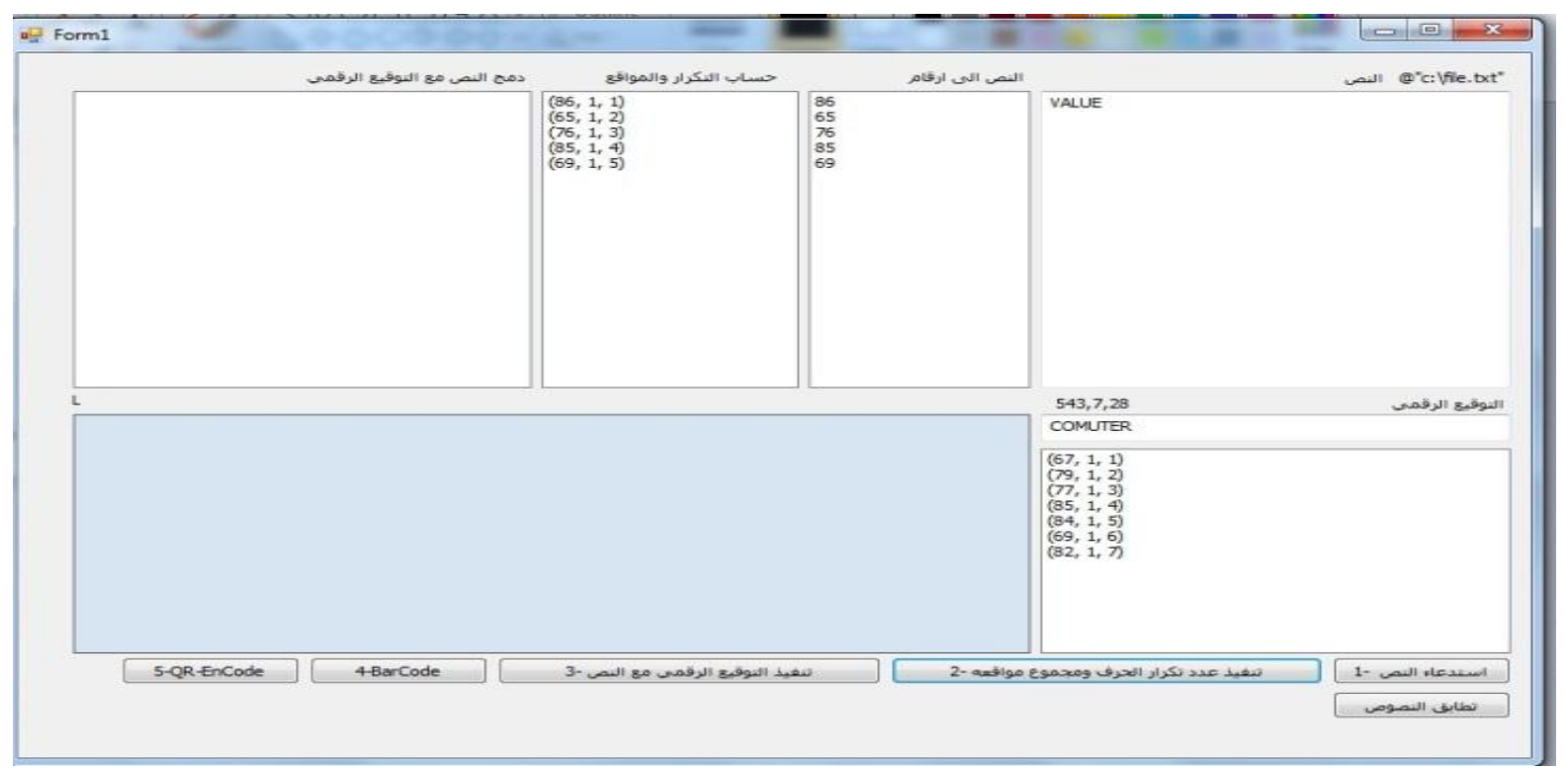

\section{الشكل( (1 - )}

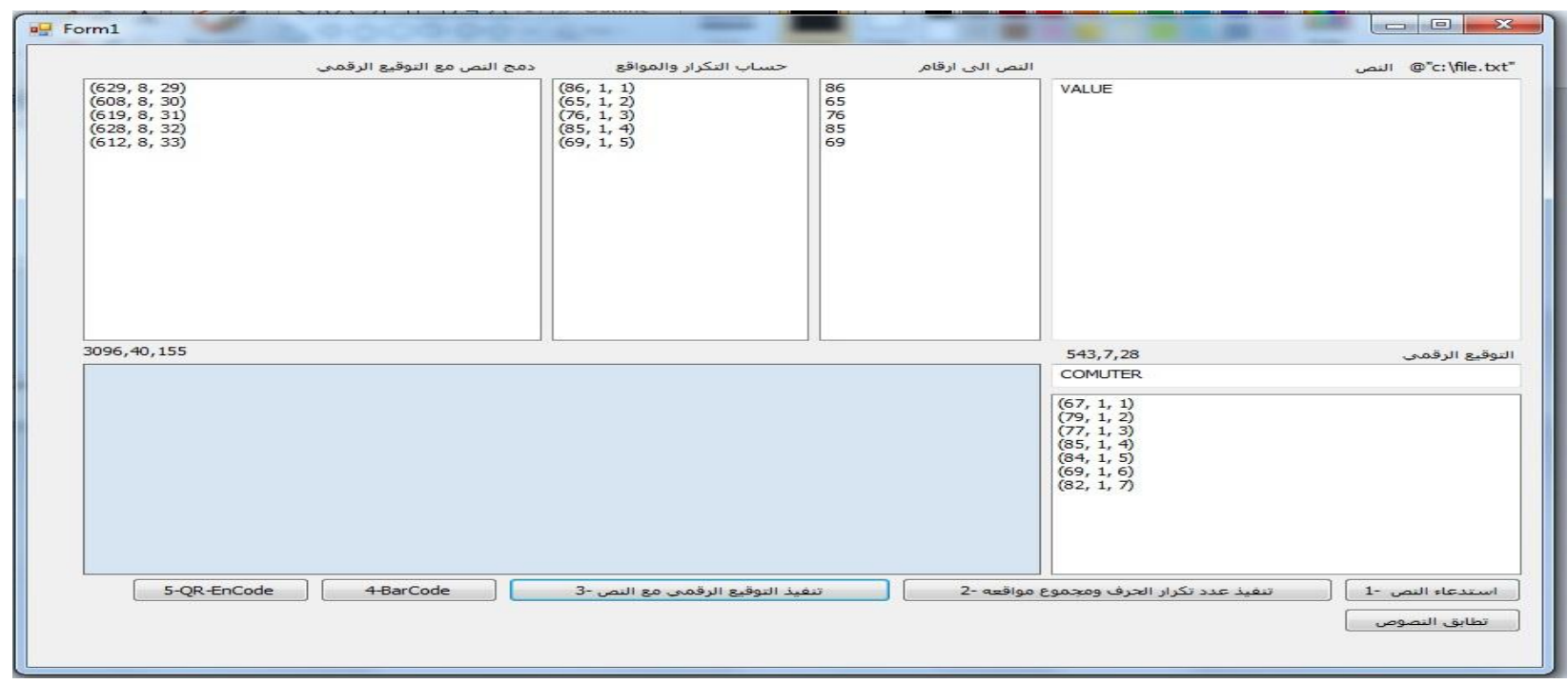

الشكل(V- 
Journal of University of Babylon for Pure and Applied Sciences,Vol.(26), No.(7): 2018

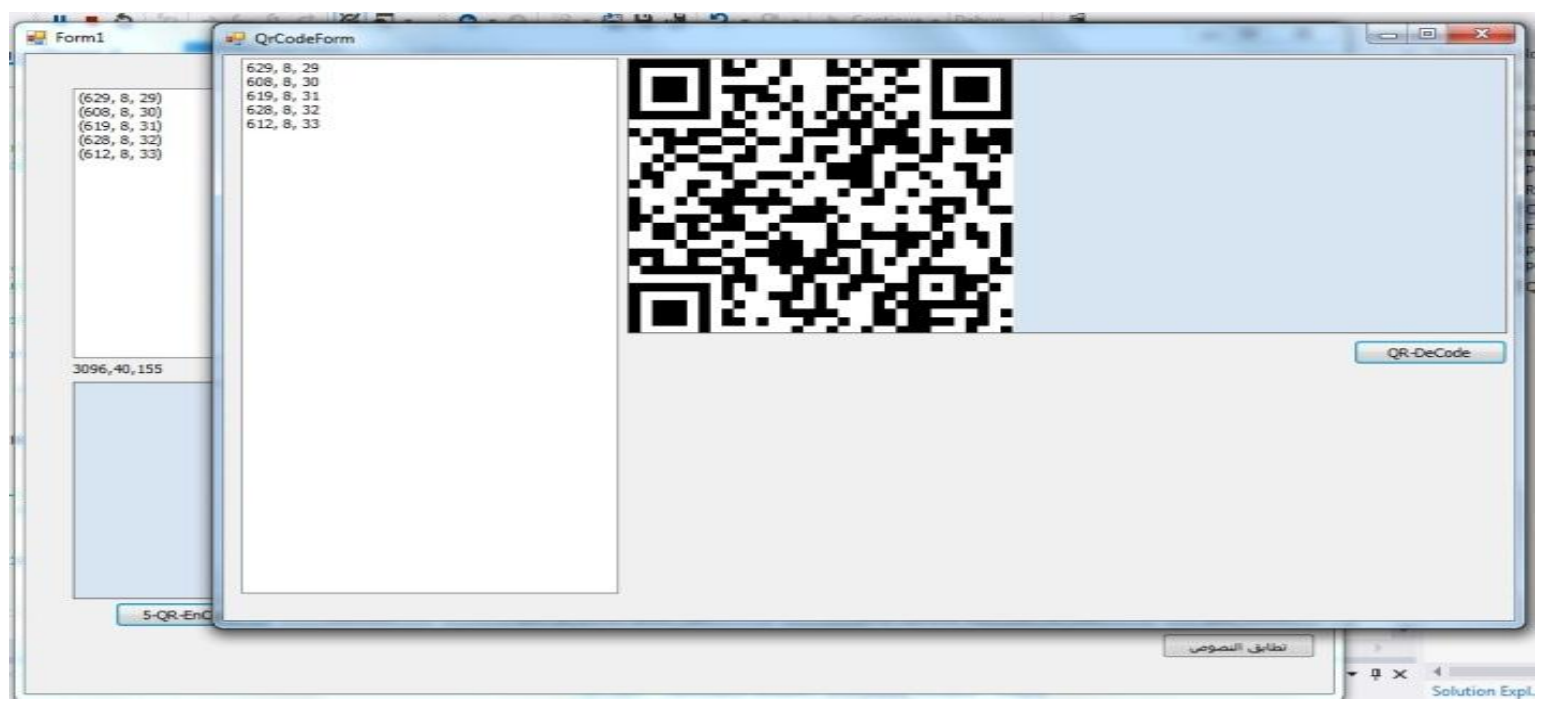

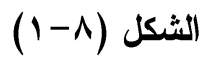

الان لو نلاحظ ان هنالك قيم رقمية تغيرت مكانها ,و لاثبات ذلك نجري الخطوات التالية.مرحلة المطابقة ل QR-Code المتكون للكلمتين (VALUE) و (VALEU) كما في الاشكال التالية.

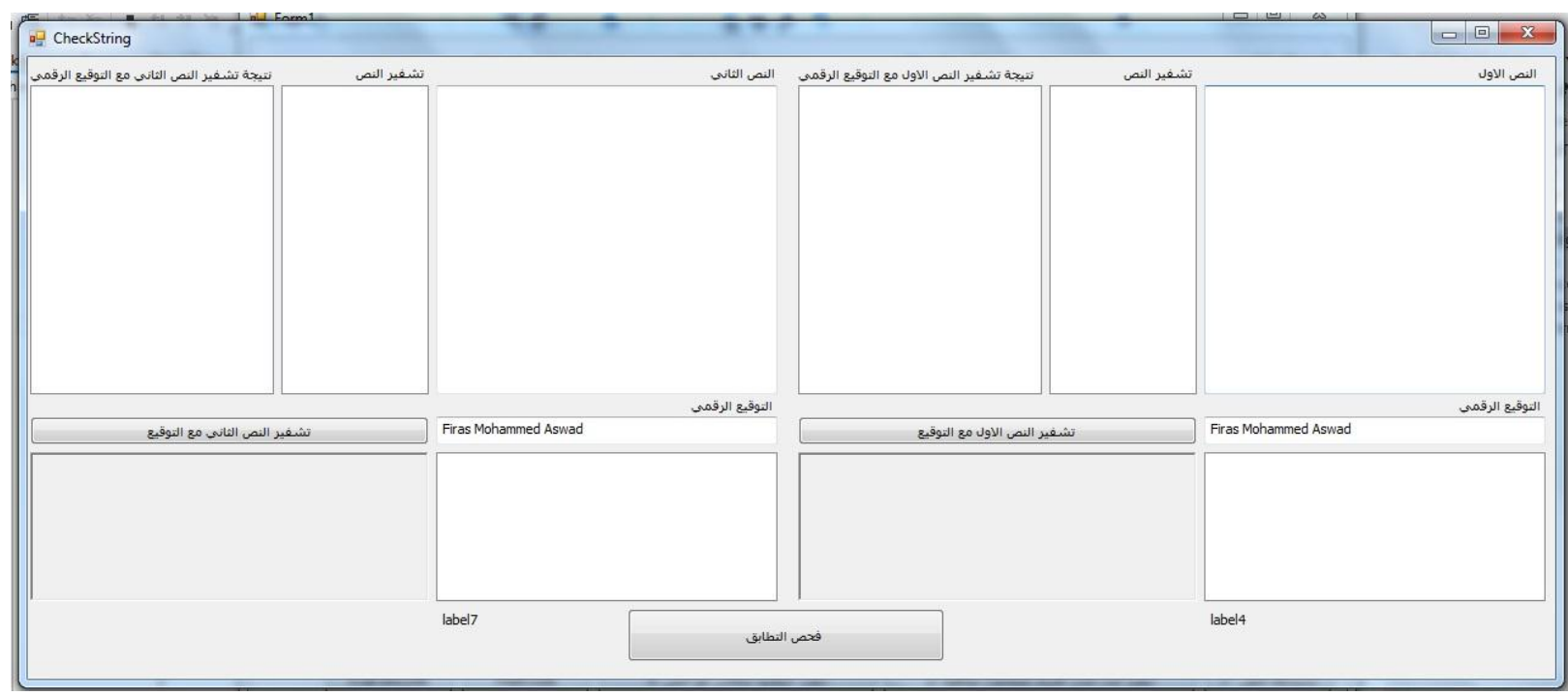

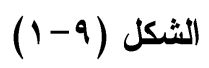

نكتب كلمة (VALUE) في مربع النص الاول , وكلمة (VALEU) في مربع النص الثاني كما في الثكل (9-1) اعلاه. سينتج QR-Code لكل كلمة كما موضح في الثكل (• (-1) التالي 


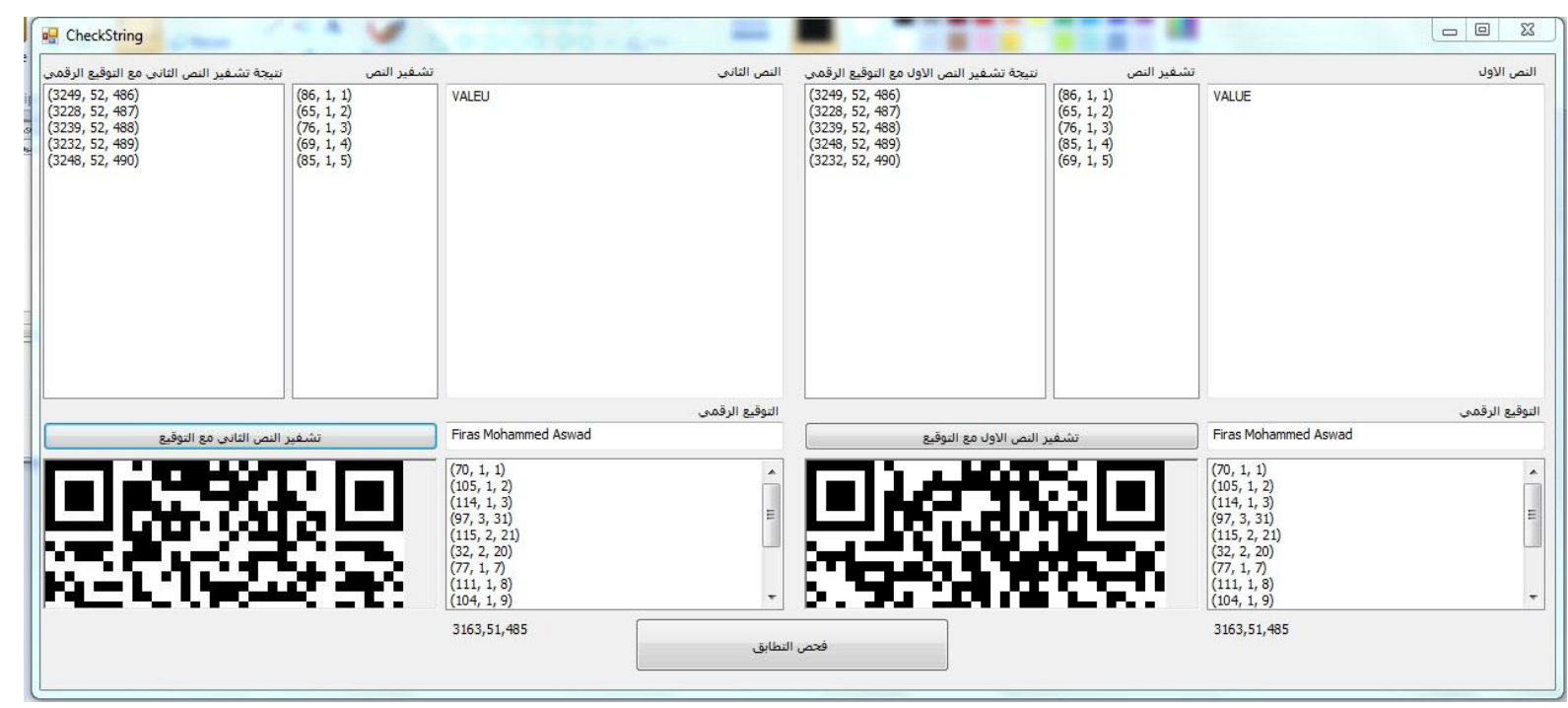

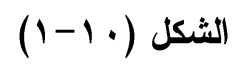

نضغط على مفتاح فحص التطابق وستضهر النتيجة كما في الثكل (11-1) التالي.

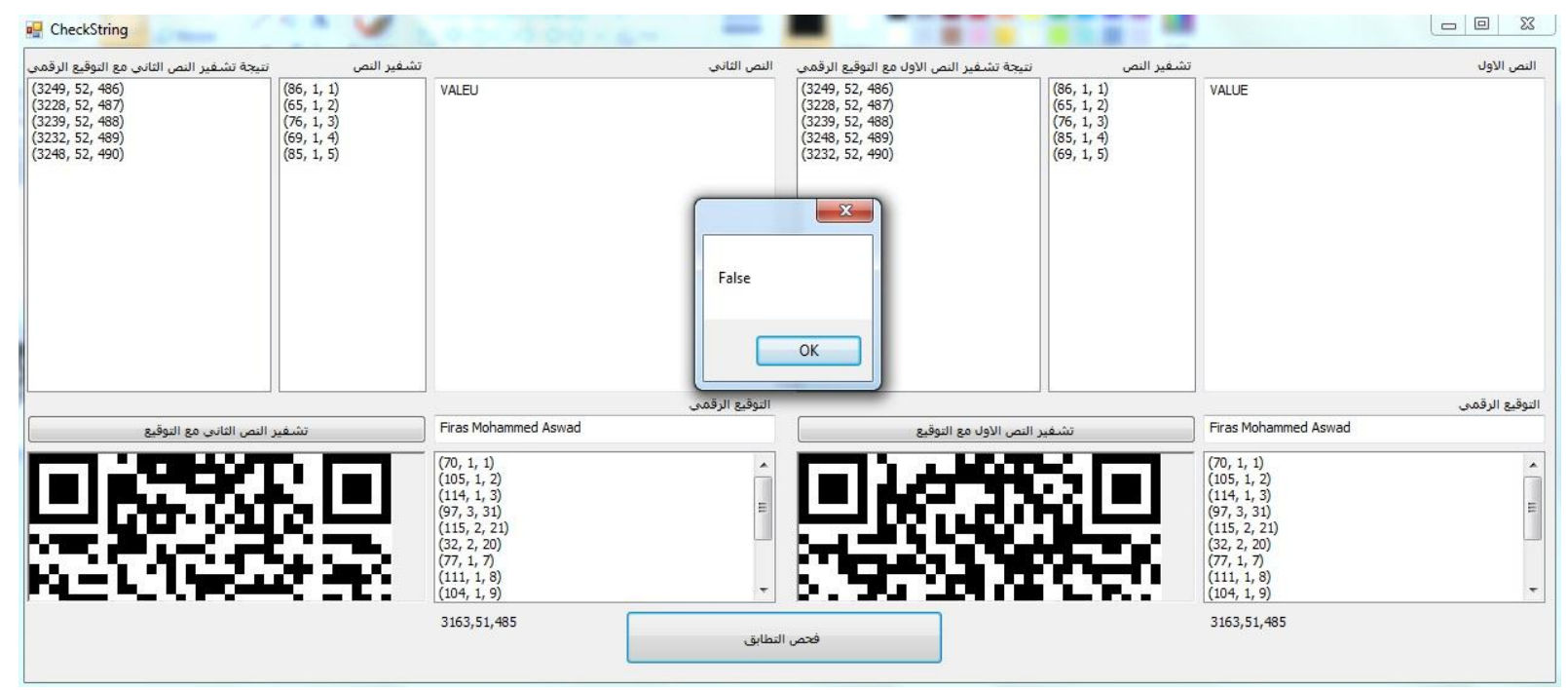

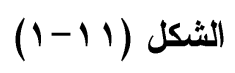

هنا لو نلاحظ نتيجة الفحص هي (False) بالرغم ان الاختلاف هو فقط تغيير مكان حرفين ضمن النص فقط.و هذا يثبت قوة ودقة هذه الخو ارزمية المستخدمة لاثبات سلامة محتوى الرسائل و اثبات اصالة المرسل عن طريق التوقيع الرقمي.هذا هو كود برنامج في لغة سي \# لتحويل الارقام الى كيو ار كود 
private string QRcodeString; public QrCodeForm(string qrcodeString)

$\{$ this.QRcodeString = qrcodeString; InitializeComponent();\}

private void QrCodeForm_Load(object sender, EventArgs e)

$\left\{\right.$ using (SaveFileDialog sfd $=$ new SaveFileDialog() \{ Filter = "Jpge $\left.\right|^{*}$.jpg", ValidateNames $=$ true $\}$ )

$$
\{\text { if }(\text { sfd.ShowDialog }()==\text { DialogResult.OK })\{
$$

MessagingToolkit.QRCode.Codec.QRCodeEncoder enCode $=$ new

MessagingToolkit.QRCode.Codec.QRCodeEncoder(); enCode.QRCodeScale = 8;

Bitmap bmp = enCode.Encode(QRcodeString); pictureBox1.Image = bmp;

bmp.Save(sfd.FileName, ImageFormat.Jpeg); \}

هنا لو نلاحظ ان حجم الكيو ار كود المنتج من الارقام مثل برقم 1 في الكود8 = enCode.QRCodeScale;); حيث يمكن ان نجعلهة اكبر بتغير رقم 1 الى رقم اكبر.

ا-هذه الطريقة الترميزية هي طريقة مثالية الى حدا ما بكثف التلاعب ان حصل في محتوى الرسائل حتى وان كان هذا التلاعب على مستوى بسيط متل تغيير مكان رمز برمز اخر ظمن الرسالة الواحدة. r- التأكد من اصالة المرسلة للرسألة بأستخدام التوقيع الرقمي.

ا-نوصي مؤسسات الحوكمة الالكترونية ,مؤسسات التجارة الاككترونية, ومؤسسات المالية الالكترونية بأعتماد هذه التقنية في مر اسلاتها لمثاليتها بكثف اي خطأ او تلاعب بالرسائل و امكانياتها العالية في اثبات اصالة المرسل . r-نوصي الباحثين بتطوير هذه التقنية وجعلها تعمل على الرسائل غير الرسائل باللغة العربية والانكليزية. ץ-اعتماد هذه التقنية لتكون بمثابة ختم مؤسسة الكترونية بستخذم في مر اسلات هذه المؤسسات. 


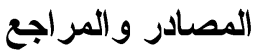

1- م.د.خلود هادي الربيعي, نهاد عبد اللطيف عبد الكريم (با • ץ) "أمن وسرية المعلومات وأثزها على الاداء التتافي دراسة تطبيقية في شركتي التأمين العر اقية العامة و الحمر اء للتأمين الاهلية ," مجلة دراسات محاسبية و مالية صفحة (0-7).

r-أ.د.علاء حسين الحمامي, د.محمد علاء الحمامي(V. . r), تكنلوجيا امنية المعلومات و انظمة الحماية,الطبعة الاولى, دار و ائل

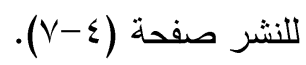

ب-أ.د.علاء حسين الحمامي, د.محمد علاء الحمامي(^ . . r),الكتابة المخفية و العلامات المائية,مكتبة الجامعة الثارقة صفحة (r-؟).

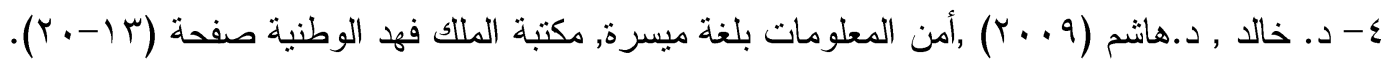

5- Partiksha M., Nitin I. "A desktop application of QR code for data security and authentication Computation Technologies (ICICT), International Conference on (2016) page (1-2).

$$
\text { 6- تعريف لغة \#C متاح على هذا الر ابط }
$$

https://ar.wikipedia.org/wiki/\%D8\%B3\%D9\%8A \%D8\%B4\%D8\%A7\%D8\%B1\%D8\%A8 